\title{
Indeterminacy and the onset of motion in a simple granular packing
}

\author{
Sean McNamara, Ramón García-Rojo, and Hans Herrmann \\ Institut für Computerphysik, Universität Stuttgart, 70569 Stuttgart, Germany \\ (Received 25 August 2004; revised manuscript received 19 May 2005; published 19 August 2005)
}

\begin{abstract}
We examine the relatively simple problem of a disk placed in a symmetric V-shaped channel, and subjected to gravity and a torque. We obtain analytic predictions of the contact forces and disk motion using two different models. In the first model, the disk is assumed to be perfectly rigid, leading to force indeterminacy. In the second model, the disk is assumed to interact with the walls of the channel via linear springs, leading to a unique solution for the contact forces. The results of these two models are compared. It is shown that there are two possible ways motion can occur-through the appearance of a null eigenvector of the stiffness matrix, or through an instability. When motion occurs through an instability, the first model cannot predict when the disk will rotate; it is necessary to know the undetermined forces in order to predict the motion of the disk. It is also shown how indeterminacy in the first model is linked to memory in the second. The analytical results are also compared with numerical simulations using two different methods, each related to one of the models.
\end{abstract}

DOI: 10.1103/PhysRevE.72.021304

PACS number(s): 45.70.-n

\section{INTRODUCTION}

\section{A. Motivation}

The quasistatic behavior of granular materials has been studied for many years by engineers seeking to provide stable foundations for buildings and roads. They typically use continuum equations, with appropriate constitutive relations, to predict the deformation of soils when a load is applied to them. Recently, there has been a great deal of work to relate these continuum equations to the micromechanicswhat is happening at the level of individual grains or contacts. One hopes to obtain a deeper physical understanding of "granular solids," as well as to suggest new continuum approaches. One powerful tool employed in this quest is numerical simulation, which gives access both to the macroscopic, continuumlike behavior of the granular material, and also to very detailed microscopic information. However, such simulations always involve approximations and various mathematical subtleties.

One of the most intriguing issues raised by numerical simulations is the indeterminacy of packings of rough, perfectly rigid particles. Since the Young's modulus of rock, glass, and metal is much greater than the stresses applied to granular materials, it is tempting to idealize the grains as infinitely hard bodies. When this is done, the problem of determining contact forces in mechanical equilibrium is indeterminate: there are usually many possible solutions [1-5]. Several questions arise: How are the different solutions related to one another? Can indeterminacy cause wrong predictions of particle motion to be given? In a model without indeterminacy, are all possible solutions accessible? What determines which one chosen?

The goal of this paper is to study the mathematical properties of two different models of granular material. One model assumes perfectly rigid particles and exhibits indeterminacy, and the other assumes the particles are deformable and produces a unique solution. Both models can be solved analytically when applied to a very simple problem. The results of the two models are then compared, giving precise answers to the questions posed in the proceeding paragraph. Furthermore, our calculations are done using formalisms then can be generalized to many particle packings. We anticipate therefore that our findings in this paper are suggestive of what happens in larger, more complicated systems.

\section{B. Synopsis}

The granular "packing" shown in Fig. 1 is perhaps the simplest system that exhibits some effects that we wish to study. The disk is subjected to a gravitational acceleration $g$ and a torque $\tau>0$. The contacts between the disk and the wall are assumed to be noncohesive and frictional, with Coulomb friction ratio $\mu$. We investigate two questions: How do the contact forces at contacts $\alpha$ and $\beta$ change in response to the imposed forces? At what value of the torque does the particle begin to rotate?

Similar problems have been investigated in at least two other cases. Ref. [5] investigated a rod wedged between two converging walls. Indeed, this work can be considered as a deeper and more precise investigation of the ideas presented in that paper. The system we study is also very close to the disk placed into an asymmetric groove considered in Ref.

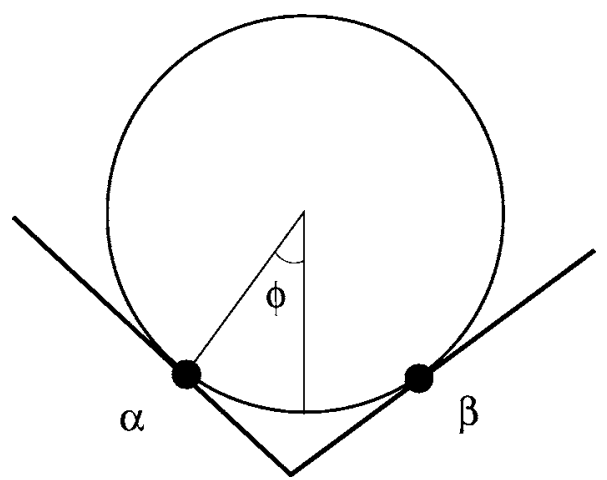

FIG. 1. A disk supported by two walls through contacts $\alpha$ and $\beta$. The angle $\phi$ suffices to characterize the geometry. 
[6]; however, the perspective taken in this paper is different. Instead of investigating a simple physical problem as realistically as possible, as in Ref. [6], this paper is concerned with the mathematical properties of two different models in the hope of gaining insight into the behavior of many particle systems. These models involve idealizations (for example, a linear force law is used at contacts, the disk is perfectly round) whose effects are not analyzed here. We believe it is useful first to understand these simple models before turning to the complexities introduced by lifting these idealizations.

We first examine some general considerations: the equations of static equilibrium, and the status of the contacts. Then we attempt to deduce the contact forces from these considerations. We show that for certain geometries and torques, one cannot decide whether the disk rotates or not. We then present a series of contact dynamics (CD) [7] and molecular dynamics (MD) [8] simulations, and point out the difference between them. Then we formulate a second method of calculating the contact forces, assuming they arise from small deformations of the particles. This second method explains all the features of the MD simulations, and sheds light on the questions mentioned above.

\section{RIGID PARTICLES}

\section{A. Static equilibrium}

The equations of static equilibrium for the disk in Fig. 1 are

$$
\begin{gathered}
R_{\alpha} \sin \phi+T_{\alpha} \cos \phi-R_{\beta} \sin \phi+T_{\beta} \cos \phi=0 \\
R_{\alpha} \cos \phi-T_{\alpha} \sin \phi+R_{\beta} \cos \phi+T_{\beta} \sin \phi=m g \\
r T_{\alpha}+r T_{\beta}=-\tau .
\end{gathered}
$$

Here, $R_{\alpha}$ indicates the normal force at contact $\alpha$, with $R_{\alpha}$ $>0$ for repulsion. $T_{\alpha}$ is the tangential force, with $T_{\alpha}>0$ when this force exerts a positive torque on the disk. $R_{\beta}$ and $T_{\beta}$ are defined in the same way.

These equations can be written in matrix form:

$$
\mathbf{c F}+\mathbf{f}_{\mathrm{ext}}=\mathbf{0}
$$

We call $\mathbf{c}$ the contact matrix:

$$
\mathbf{c}=\left(\begin{array}{cccc}
\sin \phi & \cos \phi & -\sin \phi & \cos \phi \\
\cos \phi & -\sin \phi & \cos \phi & \sin \phi \\
0 & r & 0 & r
\end{array}\right)
$$

Note that the dimensions of $\mathbf{c}$ require that it have at least one null eigenvalue. The contact forces $\mathbf{F}$ and the external forces $\mathbf{f}_{\text {ext }}$ are

$$
\mathbf{F}=\left(\begin{array}{c}
R_{\alpha} \\
T_{\alpha} \\
R_{\beta} \\
T_{\beta}
\end{array}\right), \quad \mathbf{f}_{\mathrm{ext}}=\left(\begin{array}{c}
0 \\
-m g \\
\tau
\end{array}\right) .
$$
$\mathrm{R}^{4}$ :

It is convenient to use the following orthogonal basis of

$$
\begin{aligned}
& \mathbf{F}_{x}=\frac{1}{2}\left(\begin{array}{c}
\sin \phi \\
\cos \phi \\
-\sin \phi \\
\cos \phi
\end{array}\right), \quad \mathbf{F}_{y}=\frac{1}{2}\left(\begin{array}{c}
\cos \phi \\
-\sin \phi \\
\cos \phi \\
\sin \phi
\end{array}\right) \\
& \mathbf{F}_{\theta}=\frac{1}{2}\left(\begin{array}{c}
-\cos \phi \\
\sin \phi \\
\cos \phi \\
\sin \phi
\end{array}\right), \quad \mathbf{F}_{0}=\frac{1}{2}\left(\begin{array}{c}
\sin \phi \\
\cos \phi \\
\sin \phi \\
-\cos \phi
\end{array}\right)
\end{aligned}
$$

If $\mathbf{F}$ is written in this basis:

$$
\mathbf{F}=a_{x} \mathbf{F}_{x}+a_{y} \mathbf{F}_{y}+a_{\theta} \mathbf{F}_{\theta}+a_{0} \mathbf{F}_{0}
$$

then one can verify that

$$
\mathbf{c} \mathbf{F}=\left(\begin{array}{c}
a_{x} \\
a_{y} \\
a_{x} r \cos \phi+a_{\theta} r \sin \phi
\end{array}\right)=-\mathbf{f}_{\mathrm{ext}} .
$$

This equation enables one to easily construct solutions to Eq. (2), for one has immediately $a_{x}=0, a_{y}=m g$, and $a_{\theta}=$ $-\tau /(r \sin \phi)$. However, $a_{0}$ cannot be determined in this way because $\mathbf{F}_{0}$ is a null eigenvector of $\mathbf{c}$. This is an expression of the indeterminacy in the problem.

\section{B. Contact status}

Of course, one does not have absolute freedom to choose $a_{0}$, because the following conditions must be satisfied at the contacts:

$$
R \geqslant 0, \quad \mu R \geqslant|T|
$$

where the constant $\mu$ is the Coulomb friction ratio. The first inequality excludes cohesive forces, and the second requires that the tangential forces not exceed a certain threshold.

At this point, it is useful to introduce the concept of contact status. If we have equality in the second condition in Eq. (8), the contact is said to be sliding, otherwise it is nonsliding. The tangential relative motion $v_{t}$ must be consistent with the contact status. If the contact is nonsliding, no tangential motion is allowed, but if the contact is sliding, then the tangential force must oppose the motion. Note that $v_{t}=0$ is allowed under all circumstances, so that contacts in static equilibrium can be sliding.

To see how this limits the possible values of $a_{0}$, one can first use the definition of $\mathbf{F}$ in Eq. (4) and the basis in Eq. (5) to compute the forces in terms of $\mu, \phi$, and the coefficients in the expansion of Eq. (6). Then one can use Eq. (7) to eliminate all the coefficients except for $a_{0}$. Finally, one forms the inequalities $\mu R_{\alpha} \geqslant-T_{\alpha}, \mu R_{\alpha} \geqslant T_{\alpha}, \mu R_{\beta} \geqslant-T_{\beta}$, and $\mu R_{\beta}$ $\geqslant T_{\beta}$. These yield 


$$
\begin{aligned}
& a_{0}(\mu \tan \phi+1)+\left(m g+\frac{\tau}{r \sin \phi}\right)(\mu-\tan \phi) \geqslant 0, \\
& a_{0}(\mu \tan \phi-1)+\left(m g+\frac{\tau}{r \sin \phi}\right)(\mu+\tan \phi) \geqslant 0, \\
& a_{0}(\mu \tan \phi-1)+\left(m g-\frac{\tau}{r \sin \phi}\right)(\mu+\tan \phi) \geqslant 0, \\
& a_{0}(\mu \tan \phi+1)+\left(m g-\frac{\tau}{r \sin \phi}\right)(\mu-\tan \phi) \geqslant 0 .
\end{aligned}
$$

Some of these conditions are redundant. For example, Eq. (9c) implies Eq. (9b). To see this, note that due to our choice $\tau>0$,

$$
\left(\frac{2 \tau}{r \sin \phi}\right)(\mu+\tan \phi) \geqslant 0 .
$$

Adding this to Eq. (9c) gives Eq. (9b). Thus Eq. (9b) never needs to be considered; it will be satisfied as long as Eq. (9c) is. Similar reasoning can be used to show that Eq. (9a) is redundant when $\mu-\tan \phi>0$, and that Eq. (9d) is redundant when $\mu-\tan \phi<0$. When $\mu=\tan \phi$, these conditions are equivalent.

The conditions in Eqs. (9) put limits on the value that $a_{0}$ can attain [4]. Furthermore, when a contact is sliding, we have equality in one of the above cases, and $a_{0}$ is determined: indeterminacy disappears.

\section{Application}

We now attempt to deduce the contact forces and particle motion, using only the considerations presented above. No relation between particle deformation and contact forces is assumed. Note that the biggest difficulty will be determining the coefficient $a_{0}$; the other three coefficients in the expansion in Eq. (6) can be read off from Eq. (7). The unknown value of $a_{0}$ is an expression of the indeterminacy of the problem. We must consider three separate cases, depending on the relation of $\mu$ to the slope of the sides of the groove, $\tan \phi$.

\section{Shallow slopes: $\tan \phi<\mu$}

Let us first restrict ourselves to $\tan \phi<\mu$ and $\mu<1$. Under these assumptions, we have $\mu-\tan \phi>0$, so the $a_{0}$ is constrained by the conditions Eqs. (9c) and (9d). Furthermore, $\mu \tan \phi-1<0$, so that Eq. (9c) sets a lower, and Eq. (9d) an upper bound on $a_{0}$ :

$$
a_{\min } \leqslant a_{0} \leqslant a_{\max }
$$

where

$$
a_{\min }=-\left[m g-\frac{\tau}{r \sin \phi}\right]\left(\frac{\mu-\tan \phi}{1+\mu \tan \phi}\right),
$$

$$
a_{\max }=\left[m g-\frac{\tau}{r \sin \phi}\right]\left(\frac{\mu+\tan \phi}{1-\mu \tan \phi}\right) .
$$

The quantities in the curved brackets are always positive, while the quantity in the square brackets is positive for $\tau$ $=0$, and decreases as $\tau$ increases. It vanishes when

$$
\tau=\tau_{1} \equiv m g r \sin \phi,
$$

and becomes negative when $\tau>\tau_{1}$. When it is negative, no solution for $a_{0}$ exists, for $a_{\min }>a_{\max }$. Thus there are no solutions of the equations of static equilibrium, when $\tau>\tau_{1}$ that also satisfy Eq. (8), and the disk must move. On the other hand, when $\tau<\tau_{1}$, an infinite number of such solutions exist, one for each value of $a_{0}$ satisfying Eq. (11). Finally, at $\tau=\tau_{1}$, we have $a_{\min }=a_{0}=a_{\max }=0$; hence there is a unique solution.

We have shown that the disk must rotate when $\tau>\tau_{1}$, and that static solutions exist for $\tau \leqslant \tau_{1}$, but we have not yet shown that the disk will not move when $\tau \leqslant \tau_{1}$. After all, for some choice of $a_{0}$, it might be possible to put the disk in motion. But this can be shown to be impossible by considering what happens at $\tau=\tau_{1}$. In this situation, we have $a_{0}$ $=0$, so the contact forces are

$$
R_{\alpha}=m g \cos \phi, \quad T_{\alpha}=-m g \sin \phi, \quad R_{\beta}=T_{\beta}=0 .
$$

Thus the disk is entirely supported by contact $\alpha . R_{\alpha}$ and $T_{\alpha}$ cancel the gravitational force, and $\tau$ balances the torque $r T_{\alpha}=-\tau_{1}$ exerted on the disk by the contact force. A small increase in $\tau$ and $T_{\alpha}$ will cause the disk to roll upward to the left, out of the groove. On the other hand, if $\tau<\tau_{1}$, then the torque $r T_{\alpha}=-\tau_{1}$ will not be balanced, and the disk will try to roll down the slope. Therefore, if $\tau<\tau_{1}$, the disk cannot rotate.

Another possibility is that the disk rotate in place. But this is impossible, when $\tan \phi<\mu$, because this requires $T_{\alpha}=$ $-\mu R_{\alpha}$ and $T_{\beta}=-\mu R_{\beta}$, i.e., contacts $\alpha$ and $\beta$ must be sliding. This occurs when we have equality in both Eqs. (9a) and (9c). But this cannot happen because when equality holds in Eq. (9a), then Eq. (9d) is violated.

Therefore, the value of $a_{0}$ has no effect on the motion of the disk. If $\tau<\tau_{1}$, the disk remains in the groove, although the forces cannot be uniquely determined. At $\tau=\tau_{1}$, there is a unique solution for the forces: contact $\beta$ opens and the particle's weight is supported by contact $\alpha$. For $\tau>\tau_{1}$, the disk rolls out of the groove.

Note that there is a relationship between the onset of motion and the disappearance of indeterminacy: we have motion for $\tau>\tau_{1}$ and indeterminacy for $\tau<\tau_{1}$. Indeed, we could have anticipated this when we wrote down Eqs. (9). For a disk to move, at least one contact must be sliding (or open), leading to an equality in at least one of Eqs. (9). Then this equality can be used to determine $a_{0}$, eliminating the indeterminacy.

\section{Intermediate slopes: $\mu<\tan \phi<1 / \mu$}

Now let us consider intermediate angles $\mu \leqslant \tan \phi \leqslant 1 / \mu$. At these values of $\phi$, the relevant conditions from Eqs. (9) are Eqs. (9a) and (9c). Equation (9c) sets an upper, and Eq. 
(9a) a lower, bound on $a_{0}$. Thus we have Eq. (11) again, except now

$$
\begin{aligned}
& a_{\min }=\left[m g+\frac{\tau}{r \sin \phi}\right]\left(\frac{\tan \phi-\mu}{1+\mu \tan \phi}\right), \\
& a_{\max }=\left[m g-\frac{\tau}{r \sin \phi}\right]\left(\frac{\tan \phi+\mu}{1-\mu \tan \phi}\right) .
\end{aligned}
$$

Again, the quantities in the curved brackets are positive for $\mu<\tan \phi<1 / \mu$. The quantities in the square brackets are equal and positive for $\tau=0$. But as $\tau$ increases, $a_{\min }$ increases while $a_{\max }$ decreases. When

$$
\tau=\tau_{2} \equiv m g r \frac{\mu}{1+\mu^{2}} \sec \phi
$$

we have $a_{\min }=a_{0}=a_{\max }=a_{2}$, where

$$
a_{2}=m g\left[1+\frac{\mu}{\tan \phi} \frac{1+\tan ^{2} \phi}{1+\mu^{2}}\right]\left(\frac{\tan \phi-\mu}{1+\mu \tan \phi}\right),
$$

and there is a unique solution for the contact forces. When $\tau>\tau_{2}$, no static solution satisfying the contact conditions exists, as $a_{\min }>a_{\max }$.

Furthermore, we can show that the disk cannot move when $\tau<\tau_{2}$. The disk cannot roll out of the groove, because the forces in Eq. (14) violate $\left|T_{\alpha}\right| \leqslant \mu R_{\alpha}$. If the disk is to rotate in place, we must have $T_{\alpha}=-\mu R_{\alpha}$ and $T_{\beta}=-\mu R_{\beta}$, i.e., we must have equality in Eqs. (9a) and (9c). But we have just showed that requiring these equalities leads to $\tau=\tau_{2}$, $a_{\min }=a_{0}=a_{\max }$.

Therefore, we have exactly the same situation as in Sec. II C 1. For $\tau<\tau_{2}$, there is indeterminacy without motion, and for $\tau>\tau_{2}$ there is motion. There is a unique solution for the forces precisely at $\tau=\tau_{2}$. Furthermore, note that $\tau_{1}=\tau_{2}$ when $\tan \phi=\mu$ and when $\tan \phi=1 / \mu$.

\section{Large slopes: $\tan \phi>1 / \mu$}

At large angles $(\tan \phi>1 / \mu)$, the relevant conditions are still Eqs. (9a) and (9c) as in the previous section. But now the factor $1-\mu \tan \phi$ becomes negative, transforming Eq. (9c) into a lower bound on $a_{0}$. Equation (9a) remains a lower bound, meaning that there is no upper bound on $a_{0}$. It can be made arbitrarily large. Therefore, for any $\tau>0$, it is possible to satisfy all conditions in Eq. (9) simply by making $a_{0}$ very large. (Note that all the factors multiplying $a_{0}$ in these conditions are positive when $\tan \phi>1 / \mu$.) This means that an infinite torque can be put on the disk without causing it to rotate.

On the other hand, if $\tau=\tau_{2}$, one can obtain equality in Eqs. (9a) and (9c), meaning contacts $\alpha$ and $\beta$ are sliding. If $\tau$ is increased slightly, then the disk can start to rotate in place. Therefore, both static and moving solutions coexist for $\tau>\tau_{2}$, and we cannot decide on the basis of Eqs. (9) whether the disk rotates or not.

This remarkable situation poses two questions: First, how will the CD algorithm handle this case? This algorithm tries to use the information presented in Sec. II to deduce the motion of the particles, but we have shown that this is insuf-

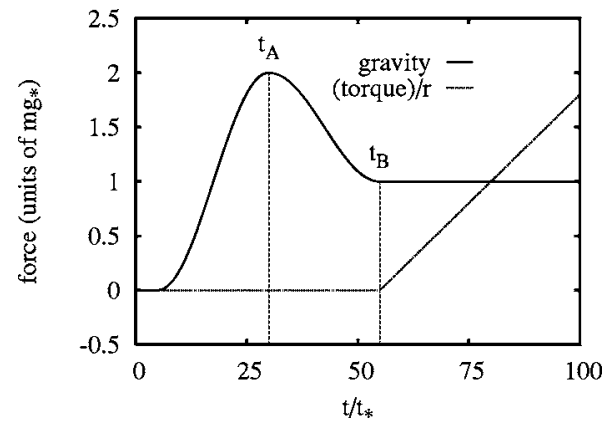

FIG. 2. The forces applied to the disk. Time is given in units of $t_{*}=\sqrt{d / g}$. The gravity attains a maximum at $t=t_{A}$, and the torque begins to increase at $t=t_{B}$.

ficient. Second, it would be very easy to construct an experiment to measure the torque needed to make the disk rotate. One could construct a groove with $\tan \phi>1 / \mu$, and place a cylinder in the groove, and try to turn the cylinder. What determines whether the cylinder rotate? These questions are investigated in the next sections.

\section{SIMULATIONS}

To verify the conclusions of the previous section, and to clarify the situation for $\tan \phi>1 / \mu$, we carried out CD and MD simulations. The disk was placed in the groove without any forces acting on it. Then gravity was turned on, and increased, until it reached a maximum of $g_{\max }$ at $t=t_{A}$. Then it was decreased, reaching $g_{*}$ at $t=t_{B}$, and thereafter was held constant. A linearly increasing torque $\tau$ was then applied until the particle began to rotate. Figure 2 shows the gravity force and torque as functions of time. In the following, we will vary $g_{\max }$, but keep $g_{*}$ fixed. In all cases, $\mu=0.5$.

The experiment can be considered as a very simple soil mechanics experiment. First, the sample, consisting of one disk, is "prepared" by pushing the disk into the groove, and "loaded" by the torque until it "yields." We are especially interested in knowing if and how the preparation affects the yielding torque.

A typical result of a simulation is shown in Fig. 3. The three nonzero coefficients $a_{y}, a_{\theta}$, and $a_{0}$ of the expansion in Eq. (6) are shown. In accordance with Eq. (7), $a_{y}$ and $a_{\theta}$ are identical for the $\mathrm{CD}$ and MD solution methods, and given by

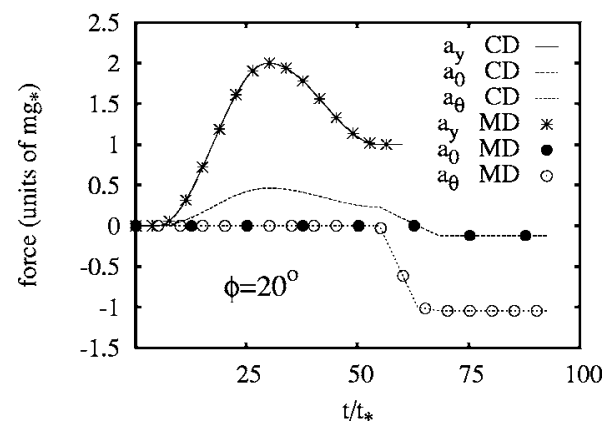

FIG. 3. The coefficients of the expansion Eq. (6) as a function of time. Here $\phi=20^{\circ}$ and $\mu=0.5$ so that $\tan \phi<\mu$. 


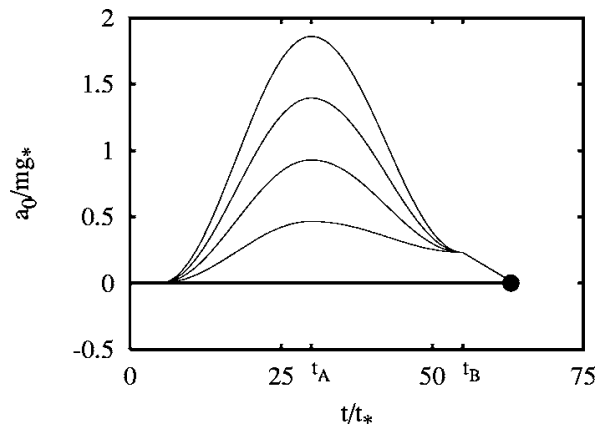

FIG. 4. The coefficient $a_{0}$ as a function of time for various values of $g_{\max } / g_{*}$. Here $\phi=20^{\circ}$ and $\mu=0.5$, so that $\tan \phi<\mu$. The thin lines are the CD simulations, with $g_{\max } / g=2$ (lowest curve), 4 , 6 , and 8 (highest curve). The thick lines are the corresponding MD simulations, which fall on top of each other in this case. The large dot indicates the point where the disk begins to rotate.

$a_{y}=m g, a_{\theta}=-\tau /(r \sin \phi)$. The coefficient $a_{0}$, however, differs between the $\mathrm{CD}$ and MD solutions. In the following, we will plot only $a_{0}$, as the other coefficients are always uniquely determined by the imposed forces. Three different values of $\phi$ will be considered, corresponding to the three subsections of Sec. II C.

\section{A. Shallow slopes: $\tan \phi<\mu$}

In Fig. 4, we plot $a_{0}$ as a function of time when $\phi=20^{\circ}$ $(\tan \phi<\mu)$, for the MD and CD simulations. In the MD simulations, $a_{0}=0$, independent of time and of $g_{\max }$. In the CD simulation, $a_{0}$ is proportional to the gravity for $t<t_{B}$. Note that the values of $a_{0}$ are significant-almost twice the weight of the particle for $g_{\max } / g_{*}=8$. However, at $t=t_{B}$, all CD simulations have the same value of $a_{0}$. As the torque is applied, $a_{0}$ moves linearly towards 0 . This occurs because when $\tau \leqslant \tau_{1}$, the disk cannot move, but $a_{0}$ is constrained between the two values given in Eq. (12), which approach each other as $\tau$ approaches $\tau_{1}$. Indeed, the diagonal line segment near $50<t / t_{*}<60$ traced out by the CD simulations corresponds to requiring equality in Eq. (9d). The conditions Eqs. (9c) and (9d) act as a "funnel" that guides $a_{0}$ toward 0 as the torque increases, so that $a_{0}=0$ when $\tau=\tau_{1}$. Thus the MD and CD methods both predict that the disk moves at $\tau$ $=\tau_{1}$. However, the two methods predict different routes to failure. MD predicts that both contacts remain nonsliding until the force at $\beta$ vanishes, and the disk starts to roll. On the other hand, $\mathrm{CD}$ predicts that the contact at $\beta$ first becomes sliding, and then later starts to roll.

\section{B. Intermediate slopes: $\mu<\tan \phi<1 / \mu$}

In Fig. 5, we show $a_{0}$ for the case of $\phi=50^{\circ}(\mu<\tan \phi$ $<1 / \mu)$. The behavior of the CD simulation is quite similar to the preceding case. For $t<t_{B}, a_{0}$ is again proportional to gravity, but attaining even larger values than before. At $t$ $=t_{B}$, all CD simulations are identical, and they evolve together during the loading. They again encounter the "funnel" arising from the lower and upper bounds on $a_{0}$ given in Eq.

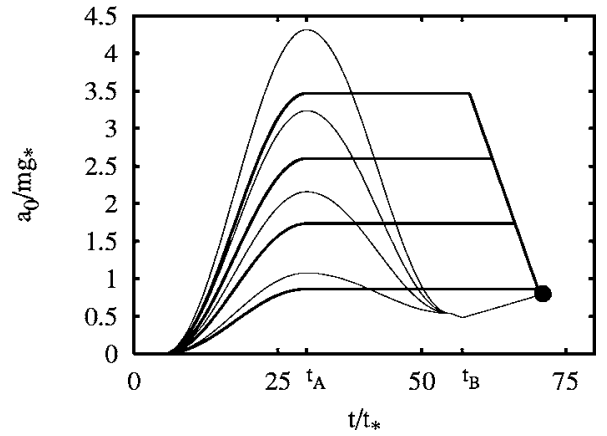

FIG. 5. The undetermined coefficient $a_{0}$ from Eq. (6) as a function of time. Here, $\phi=50^{\circ}$ and $\mu=0.5$, so that $\mu<\tan \phi<1 / \mu$. The thin lines are the CD simulations, with $g_{\max } / g=2$ (lowest curve), 4 , 6 , and 8 (highest curve). The thick lines are the MD simulations with the same values of $g_{\max } / g$. The large dot indicates the point where the disk begins to rotate.

(15), and move toward $a_{0}=a_{2}$, and the disk starts to rotate when $\tau=\tau_{2}$.

The MD simulation has a quite different behavior. For $t$ $<t_{A}, a_{0}$ is also proportional to the gravity, although lower than the $\mathrm{CD}$ value. But for $t>t_{A}, a_{0}$ remains constant, so that $a_{0}$ is proportional to $g_{\max }$, even at $t=t_{B}$. In this case, therefore, the preparation does make a difference. The MD simulations remember the value of $g_{\max }$, and this memory is stored in $a_{0}$. This simple example shows how the indeterminacy of forces in perfectly rigid particles is related to history dependency in packings of deformable particles. The coefficient $a_{0}$, which is undetermined in the perfectly rigid case, here contains the memory of the packing, and is simply proportional to the greatest downward force that the disk has experienced in the past.

The history dependency of the MD simulation, however, does not affect the yielding torque. The different MD simulations encounter the funnel at different times, but are all guided towards the point $\tau=\tau_{2}, a_{0}=a_{2}$, where the disk begins to rotate. Therefore, the memory in the MD simulation can only be detected by inspecting the contact forces.

\section{Large slopes: $\tan \phi>1 / \mu$}

Finally, in Fig. 6, we show the case where $\phi=80^{\circ}$ $(\tan \phi>1 / \mu)$. This case has some important differences from the preceding cases. First of all, both the CD as well as MD exhibits history dependence, as the contact forces at the end of the sample preparation and the yielding torque depend on $g_{\text {max }}$. Second, only half of the funnel operates as before.

One may ask how the CD simulation can exhibit memory, because it deduces the contact forces from the principles stated in Sec. II but the history of the packing does not enter into those considerations. The memory of the CD algorithm comes from the way it chooses the contact forces. It begins with an initial guess, which it then refines through an iterative process until it arrives at a satisfactory solution. One usually takes the initial guess to be the solution of the previous time step because convergence is faster. But in Fig. 6, it is clear that this choice of initial guess also serves to make 


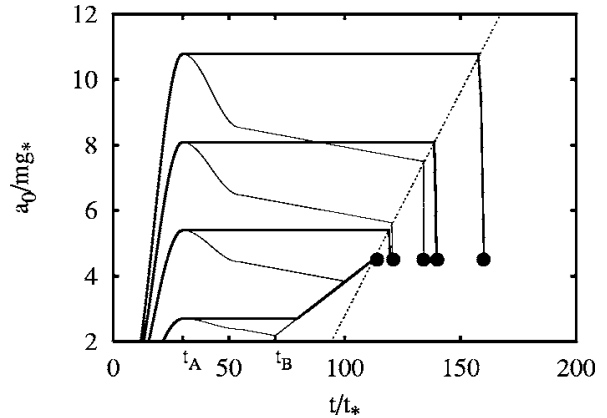

FIG. 6. Coefficients of the expansion Eq. (6) as a function of time. Here, $\phi=80^{\circ}$ and $\mu=0.5$, so that $\tan \phi>1 / \mu$. The thin lines are the CD simulations, with $g_{\max } / g=2$ (lowest curve), 4,6 , and 8 (highest curve). The thick lines are the MD simulations with the same values of $g_{\max } / g$. The large dots indicates the points where the disk begins to rotate. The diagonal dashed line is obtained by requiring equality in Eq. (9c).

the $\mathrm{CD}$ algorithm history dependent, as the solution chosen depends on the initial guess [5]. But this memory is not the same as in the MD simulations. Nor is it clear why the preparation of the sample makes a difference at $\phi=80^{\circ}$ but not at $\phi=50^{\circ}$.

But the most important difference with the previous examples is that only half of the funnel works as before. As was noted earlier, there is no longer an upper bound on $a_{0}$; Eqs. (9a) and (9c) are both lower bounds. From the figure, one can see that the lower bound set by Eq. (9a) functions as before. When $a_{0}$ reaches this lower bound, it then increases linearly as the torque is increased, until equality is obtained in both Eqs. (9a) and (9c). Then the disk begins to rotate. The behavior of the lower bound set by Eq. (9c) is quite different. When equality is obtained in that condition, the value of $a_{0}$ jumps discontinuously, and the particle begins to rotate. As a result, the yielding torque is determined by when the system first satisfies equality in Eq. (9c), and this in turn depends on the value of $a_{0}$ set by the preparation phase of the experiment. Thus in this case, the memory does affect the yielding torque.

In Fig. 7, we show the yielding torque as a function of $g_{\max }$. It is initially independent of $g_{\max }$, corresponding to the case where the system first meets the lower edge of the funnel. Then, CD and MD give different yielding torques. The MD values are well predicted by a result that will be obtained in Sec. IV D 4.

The $\mathrm{CD}$ values for the yielding torque are equal to or below the MD ones, because $a_{0}$ decreases as the torque is increased, while in the MD case, $a_{0}$ remains constant. This is plainly visible in Fig. 6. The different $C D$ points correspond to different iteration procedures. The $\mathrm{CD}$ algorithm searches for a solution by adjusting the contact forces one by one. It considers a given contact, and calculates the change in its contact forces needed to prevent interpenetration and minimize sliding. It then adds this to change to its current guess for the forces. After passing over all the contacts a certain number of times, the changes in the force needed fall below a certain threshold, and the solution is accepted. But this procedure can be altered by multiplying the calculated

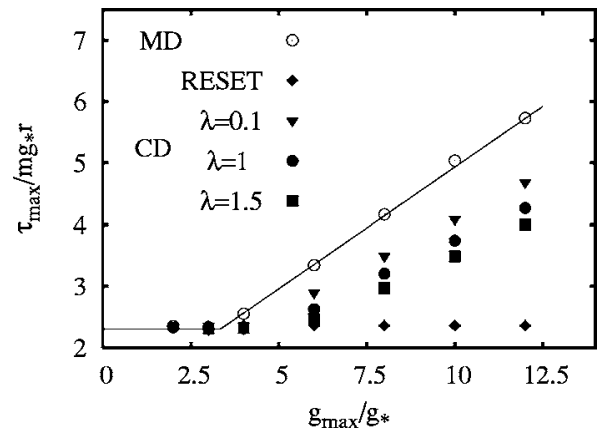

FIG. 7. The torque at which the disk begins to rotate at $\phi$ $=80^{\circ}$ and $\mu=0.5$, for different values of $g_{\max }$. The four different sets of points for $\mathrm{CD}$ correspond to the different iteration algorithms described in the text. The line shows the theoretical prediction given in Sec. IV D 4

change in forces by a number $\lambda$, with $\lambda=1$ corresponding to the usual iteration procedure. Changing $\lambda$ changes the yielding torque, as shown above. Furthermore, the memory in the $\mathrm{CD}$ algorithm can be erased at each time step by always using $\mathbf{F}=\mathbf{0}$ as the initial guess, instead of the solution of the last time step. When this is done, one obtains the points labeled "RESET" in Fig. 7 and the yielding torque is independent of $g_{\max }$. This result emphasizes the important role of memory in this experiment.

\section{DEFORMABLE PARTICLES}

\section{A. Formalism}

We now present a second method of calculating the contact forces that is able to explain all features of the MD simulations presented in the previous section. The cost of this additional information is that more assumptions must be made. First of all, one must specify how the forces depend on the deformations, but more significantly, one must specify the past history of the packing. This method shows how indeterminacy is replaced by memory. More precisely, when $\tan \phi>\mu$, we show that $a_{0}$ in Eq. (6) stores information about the largest downward force that has been exerted on the disk in the past. Indeed, $a_{0}$ is a quantification of the "degree of wedging" discussed in Ref. [5].

We model the deformations in a very simple way, assuming that contact forces are generated by springs that are stretched by the motion of the disk. Our model is inspired by the MD simulation method [8], but we are able to apply it analytically to our simple problem.

When two bodies touch, a normal and a tangential spring be created at the instant of contact. The contact forces are simply proportional to the spring lengths:

$$
R=-k \delta_{n}, \quad T=-k \delta_{t},
$$

where $\delta_{n}$ and $\delta_{t}$ are the normal and tangential spring lengths, and $k$ is the spring constant, here assumed to be equal for both the tangential and normal springs. One normally includes damping in Eq. (18), but we will assume that the motion is quasistatic, i.e., a sequence of equilibrium states. 
Under this assumption, the particle velocities are vanishingly small, and so are the damping forces. Equation (18) leads to a vector equation for $\mathbf{F}$ in terms of the spring lengths $\mathbf{D}$ :

$$
\mathbf{F}=-k \mathbf{D},
$$

where $\delta_{n, \alpha}, \delta_{t, \alpha}, \delta_{n, \beta}$, and $\delta_{t, \beta}$ are gathered into $\mathbf{D}$ just as the contact forces are arranged in $\mathbf{F}$ [see Eq. (4)].

The spring lengths obey

$$
\dot{\delta}_{n}=v_{n}, \quad \dot{\delta}_{t}=\left\{\begin{array}{l}
v_{t}, \\
\pm \mu v_{n},
\end{array}\right.
$$

where $v_{n}$ and $v_{t}$ are the normal and tangential components of the relative velocity. There are two choices for $\dot{\delta}_{t}$ because $\mu R \geqslant|T|$ means that the tangential spring has a maximum allowable length: $\left|\delta_{t}\right| \leqslant \mu \delta_{n}$. If applying $\dot{\delta}_{t}=v_{t}$ would lead to a violation of this condition, the second choice is taken. Eq. (20) relates the time derivative of $\mathbf{D}$ to the velocity of the disk. If all contacts are nonsliding, we have

$$
\begin{gathered}
\dot{\delta}_{n, \alpha}=v_{x} \sin \phi+v_{y} \cos \phi, \\
\dot{\delta}_{t, \alpha}=v_{x} \cos \phi-v_{y} \sin \phi+r \omega, \\
\dot{\delta}_{n, \beta}=-v_{x} \sin \phi+v_{y} \sin \phi, \\
\dot{\delta}_{t, \beta}=v_{x} \cos \phi+v_{y} \sin \phi+r \omega .
\end{gathered}
$$

Gathering the velocities of the disk into a single vector

$$
\mathbf{v}=\left(\begin{array}{c}
v_{x} \\
v_{y} \\
\omega
\end{array}\right),
$$

Eqs. (21) becomes

$$
\dot{\mathbf{D}}=\mathbf{c}^{T} \mathbf{v},
$$

where $\mathbf{c}^{T}$ is the transpose of $\mathbf{c}$ in Eq. (3). The appearance of $\mathbf{c}^{T}$ is not a coincidence, but a general property valid for all granular packings [9].

It remains to incorporate the status of the contacts into our formalism. This can be be done by inserting a matrix $\mathbf{S}$ that depends on the contact status into Eq. (23):

$$
\dot{\mathbf{D}}=\mathbf{S c}^{T} \mathbf{v},
$$

where

$$
\mathbf{S}=\left(\begin{array}{cc}
S_{\alpha} & 0 \\
0 & S_{\beta}
\end{array}\right) .
$$

Here, $S_{\alpha}$ is the identity matrix if contact $\alpha$ is nonsliding, $S_{\alpha}=0$ if it is open, and

$$
S_{\alpha}=\left(\begin{array}{cc}
1 & 0 \\
\pm \mu & 0
\end{array}\right)
$$

if it is sliding.

Finally, let us not forget Newton's equation of motion:

$$
\mathbf{M i v}=\mathbf{c F}+\mathbf{f}_{\mathrm{ext}} .
$$

Here, the matrix $\mathbf{M}$ contains the mass $m$ and moment of inertia $I$ of the disk on the diagonal:

$$
\mathbf{M}=\left(\begin{array}{ccc}
m & 0 & 0 \\
0 & m & 0 \\
0 & 0 & I
\end{array}\right) .
$$

Equations (19), (24), and (27) form a set of equations that can be solved for the motion of the disk. Note that $\mathbf{c}$ remains constant because all contacts are between the disk and the straight walls. The status of the contacts can change, however, so it is useful to divide time up into segments $\left[t_{0}, t_{1}\right],\left[t_{1}, t_{2}\right], \ldots$, where the changes of contact status occur at the times $t_{1}, t_{2}, \ldots$. In the interior of a time interval, the matrices c, S, and $\mathbf{M}$ are constant, so Eqs. (19), (24), and (27) can be combined into

$$
\mathbf{M} \ddot{\mathbf{v}}=-\mathbf{Q v}+\dot{\mathbf{f}}_{\mathrm{ext}},
$$

where the matrix $\mathbf{Q}=k \mathbf{c S c} \mathbf{c}^{T}$ is called the stiffness matrix and gives the changes in the forces that arise from a small displacement of the disk. The MD simulations used to obtain the results in Sec. III are numerical solutions of Eq. (29). Those simulations concerned very slow motion of the disk, and thus we make the quasistatic approximation, and assume that the disk is always in an equilibrium state. Thus the left hand side of Eq. (29) vanishes:

$$
\mathbf{Q v}=\dot{\mathbf{f}}_{\mathrm{ext}} .
$$

This is the equation that we will analyze in the rest of this paper. It is capable of explaining the results obtained in Sec. III.

\section{B. Relation to other theories}

Before proceeding, several remarks on Eq. (30) are in order. Let us begin by pointing out that the derivation of Eq. (30) can be generalized to packings with a large number of particles. In this case, the matrices $\mathbf{c}$ and $\mathbf{S}$ can be constructed [4]. It is also possible to generalize to the case where tangential and normal motions have differing stiffness, or even where each contact has different stiffness. We therefore expect that the results presented here can be generalized to packings of many particles.

Let us also note that a numerical method, sometimes called the "granular element method" [10], is based on Eq. (30). It is less common than MD and CD because one must rebuild the stiffness matrix $\mathbf{Q}$ every time a contact opens, closes, or becomes sliding. Nevertheless, it has been compared with MD simulations and used to investigate the quasistatic deformation of granular materials $[10,11]$.

Next, we point out that Eq. (30) can be written in terms of increments instead of time derivatives:

$$
\mathbf{Q} \delta \mathbf{u}=\delta \mathbf{f}_{\mathrm{ext}} .
$$

Here, $\delta \mathbf{u}$ is a small increment of displacement caused by an incremental change in the applied load $\delta \mathbf{f}_{\text {ext }}$. The difference between this equation and Eq. (30) is that all reference to 
time has been removed from Eq. (31). This is a consequence of the quasistatic approximation: since the system is always in mechanical equilibrium, it does not matter how it moves from one state to another (as long as it is done slowly enough).

Finally let us point out several parallels between Eqs. (30) and (31) and the theories of elasticity and elastoplasticity in continuum mechanics. Equation (31) resembles Hooke's law in elasticity, with $\mathbf{Q}$ playing the role of the elasticity tensor, and giving a linear relation between the forces and the displacements. However, in continuum mechanics, the forces and displacements are given by the stress and strain tensors, which are second rank tensor functions of position. In Eq. (31), the analogous quantities are $\delta \mathbf{u}$ and $\delta \mathbf{f}_{\text {ext }}$. Furthermore, as long as there are no sliding contacts, all deformations are reversible-they can be removed simply by removing the applied force, just as in elasticity.

When a contact becomes sliding, however, the analogy to elasticity breaks down, energy will be dissipated at the sliding contact, and permanent deformation will occur. However, one can then consider an analogy to elastoplasticity $[12,13]$, which was developed to describe the irreversible deformation of metals. In this theory, the stress is considered as a point in a space whose coordinates are the components of the stress tensor. As the stress changes, this point moves correspondingly through stress space. Elastoplasticity posits that the origin of stress space is enclosed by a "yield surface." As long as the system remains within the yield surface, the material behaves elastically-all deformation vanishes if the stress returns to 0 . But when the system arrives at the yield surface, the material deforms "plastically" (irreversibly) as well as elastically. As the stress increases further, the yield surface expands as well, so that the system remains always on the yield surface. If the stress is decreased, the system moves inside the yield surface, and the deformations are again elastic.

Although not obvious from Eq. (30), the description put forth here behaves in a similar way. Requiring equality in Eq. (9) defines a "yield surface" in the four-dimensional space $\left(R_{\alpha}, T_{\alpha}, R_{\beta}, T_{\beta}\right)$. As long as the system remains inside this surface, the disk's motions are reversible and elastic. Once the system arrives at this surface, irreversible motion occurs. Another parallel to elastoplasticity occurs if the motion is reversed: in this case, the sliding contact becomes nonsliding, and the deformation is again elastic. This analogy could be studied further, but this is beyond the scope of this paper.

\section{The onset of motion}

In deriving Eq. (30), we assumed that the motion is quasistatic, so that the left hand side of Eq. (29) containing the inertia of the disk could be neglected. However, we are also interested in the onset of motion, i.e., when a static configuration yields and begins to move. There are two different ways motion can begin.

The first way occurs when the stiffness matrix $\mathbf{Q}$ has a null eigenvector, that is, there is a vector $\mathbf{v} * \neq 0$ such that $\mathbf{Q v} *=0$. The motion given by $\mathbf{v}_{*}$ is called a "mechanism" [9]. When there is a mechanism, $\mathbf{Q}$ maps $\mathbb{R}^{3}$ into a two- dimensional subspace, and if $\dot{\mathbf{f}}_{\mathrm{ext}}$ is not in this subspace, Eq. (30) has no solution. This means that the inertia terms must be included, i.e., Eq. (29) must be used instead. Equation (29) always has a solution, since $\mathbf{M}$ is a diagonal matrix with positive entries, and thus maps $\mathbb{R}^{3}$ onto itself. If $t_{*}$ is the time when $\mathbf{Q}$ becomes singular (perhaps due to a change in contact status). Then for times $t$ just after $t_{*}$, the solution to Eq. (29) has the form

$$
\mathbf{v} \sim\left(t-t_{*}\right)^{2} \mathbf{M}^{-1} \dot{\mathbf{f}}_{*},
$$

where $\dot{\mathbf{f}}_{*}$ is the part of $\dot{\mathbf{f}}_{\text {ext }}$ for which no solution to Eq. (30) exists. We will call this "motion through a mechanism." It is characterized by accelerations of order the imposed external force divided by the inertia.

The second way to motion occurs when the quadratic form $\mathbf{v}^{T} \mathbf{Q v}$ becomes negative. To see this, we dot Eq. (29) through by $\mathbf{v}$ and obtain

$$
\mathbf{v}^{T} \mathbf{M} \ddot{\mathbf{v}}=-\mathbf{v}^{T} \mathbf{Q v}+\mathbf{v} \cdot \dot{\mathbf{f}}_{\text {ext }} .
$$

We must have $\mathbf{v} \cdot \dot{f}_{\text {ext }}>0$, because when the external forces are changed, the disk must move in the direction of the change in the force. In the quasistatic approximation, the left hand side of Eq. (33) is set to zero. Thus to obtain equality, we must have

$$
\mathbf{v}^{T} \mathbf{Q v}>\mathbf{0} .
$$

This inequality must hold, otherwise the quasistatic approximation cannot be made. In the context of elastoplasticity, this condition is called Hill's stability criterion [14].

Let us now investigate the motion just after Eq. (34) has been violated. As before, we let $t_{*}$ be the time when the motion begins. Just after $t_{*}$, the motion will be given by Eq. (32), since the term $\mathbf{Q v}$ requires a finite time to change. Soon, however, this term dominates the imposed forces, so that Eq. (29) becomes $\mathbf{M} \ddot{\mathbf{v}}=-\mathbf{Q v}$. The solution is then

$$
\mathbf{v} \sim \mathbf{v}_{*} e^{t \sqrt{Q / M}},
$$

where $\mathbf{v}_{*}$ is the vector for which $Q=-\left(\mathbf{v}_{*}^{T} \mathbf{Q} \mathbf{v}_{*}\right) /\left(\mathbf{v}_{*} \cdot \mathbf{v}_{*}\right)$ is a maximum, and $M=\left(\mathbf{v}_{*}^{T} \mathbf{M} \mathbf{v}_{*}\right) /\left(\mathbf{v}_{*} \cdot \mathbf{v}_{*}\right)$. Thus, when Eq. (34) is violated, $\mathbf{Q}$ acts like a negative number in Eq. (29), and the contact forces then amplify the velocities, which then grow exponentially. In this case, the packing fails catastrophically, with the contact forces changing much more quickly than $\mathbf{f}_{\text {ext }}$. We call this "motion through an instability." It is characterized by a time scale that is much shorter than motion by a mechanism.

\section{Application}

\section{Calculation of displacements}

Let us now show how the contact forces can be calculated without indeterminacy. We calculate the coefficients of the expansion in Eq. (6). We can construct an equation for these coefficients by differentiating Eq. (19) with respect to time, and combining it with Eq. (24), leading to $\dot{\mathbf{F}}=\mathbf{S c}^{T} \mathbf{v}$. Then we project this equation onto the basis given in Eq. (5) by left- 
multiplying it by a, a $4 \times 4$ matrix whose rows are the basis vectors in Eq. (5), but without the factor of 1/2. After doing this, we obtain

$$
\left(\begin{array}{c}
\dot{a}_{x} \\
\dot{a}_{y} \\
\dot{a}_{\theta} \\
\dot{a}_{0}
\end{array}\right)=k \mathbf{a S c} \mathbf{c}^{T} \mathbf{v}
$$

Equation (36) represents four equations, one corresponding to each of $a_{x}, a_{y}, a_{\theta}$, and $a_{0}$. The first three coefficients are known from Eq. (7); thus the first three equations above can be used to find the three components of $\mathbf{v}$. Then the last equation can be used to determine $\dot{a}_{0}$. The displacement of the disk is obtained by integrating $\mathbf{v}$.

\section{Shallow angles: tan $\phi<\mu$}

When $\tan \phi<\mu$, the contacts remain nonsliding until the disk begins to rotate. Therefore, we can consider the entire experiment to take place during one time interval.

When the contacts are nonsliding,

$$
k \mathbf{a S c} c^{T}=2 k\left(\begin{array}{ccc}
1 & 0 & r \cos \phi \\
0 & 1 & 0 \\
0 & 0 & r \sin \phi \\
0 & 0 & 0
\end{array}\right)
$$

Using this result in Eq. (36), we have

$$
\begin{gathered}
\dot{a}_{x}=2 k v_{x}+2 k r \omega \cos \phi, \\
\dot{a}_{y}=2 k v_{y}, \\
\dot{a}_{\theta}=2 k r \omega \sin \phi, \\
\dot{a}_{0}=0 .
\end{gathered}
$$

From the last line, we can see that $a_{0}$ remains constant as long as the contacts are nonsliding. This explains why $a_{0}$ $=0$ for all the MD simulations in Fig. 4: $a_{0}=0$ at the beginning of the simulation, and thus remains so until the disk rolls. This also explains why $a_{0}$ is constant when $g$ is decreased in Figs. 5 and 6.

Next let us calculate the disk's motion. Equations (38) can be solved for the velocities and integrated. In this way, the displacement $\mathbf{u}$ of the disk can be shown to be

$$
\mathbf{u}=\frac{1}{2 k}\left(\begin{array}{c}
0 \\
-m g \\
\tau /\left(r^{2} \sin ^{2} \phi\right)
\end{array}\right) \text {. }
$$

Because no contact changes its status, the motion is perfectly reversible. This is another way to see that the packing has no memory when $\tan \phi<\mu$.

Finally, let us analyze the onset of motion. For $\tan \phi$ $<\mu$, motion occurs through a mechanism, as can be seen by constructing the stiffness matrix $\mathbf{Q}$ at the time when the motion begins. It was shown in Sec. II C 1, Eq. (14) that the contact $\beta$ opens when the motion begins, while contact $\alpha$ remains nonsliding. The submatrices making up $\mathbf{S}$ in Eq. (25) are thus $S_{\alpha}=\left(\begin{array}{ll}1 & 0 \\ 0 & 1\end{array}\right)$ and $S_{\beta}=0$. This leads to

$$
\mathbf{Q}=k\left(\begin{array}{ccc}
1 & 0 & r \cos \phi \\
0 & 1 & -r \sin \phi \\
r \cos \phi & -r \sin \phi & r^{2}
\end{array}\right) \text {. }
$$

There is a vector $\mathbf{V}_{*} \neq \mathbf{0}$ such that $\mathbf{Q} v_{*}=\mathbf{0}$, namely,

$$
\mathbf{V} *=\left(\begin{array}{c}
-r \cos \phi \\
r \sin \phi \\
1
\end{array}\right) \text {. }
$$

The motion given by $\mathbf{v}_{*}$ is the particle rolling upward to the left out of the groove, as described in Sec. II C 1.

\section{Intermediate slopes: $\mu<\tan \phi<1 / \mu$}

When $\tan \phi>\mu$ and $t<t_{A}$, the contacts are sliding with $T_{\alpha}=-\mu R_{\alpha}$ and $T_{\beta}=\mu R_{\beta}$ :

$$
k \mathbf{a S c}^{T}=2 k \cos ^{2} \phi\left(\begin{array}{ccc}
\tan ^{2} \phi-\mu \tan \phi & 0 & 0 \\
0 & 1+\mu \tan \phi & 0 \\
-\tan \phi-\mu \tan ^{2} \phi & 0 & 0 \\
0 & \tan \phi-\mu & 0
\end{array}\right) .
$$

Changes in contact status will occur, so it is necessary to subdivide the experiment into time intervals. The first change of status occurs at $t=t_{A}$, when the derivative of the gravity changes sign, so we define our first interval to end at $t=t_{A}$. Calculating the particle displacements using the same method as before yields

$$
u_{y}\left(t_{A}\right)=-\frac{m g_{\max }}{2 k} \frac{\sec ^{2} \phi}{1+\mu \tan \phi} .
$$

as before, $u_{x}\left(t_{A}\right)=u_{\theta}\left(t_{A}\right)=0$. We also have

$$
a_{0}\left(t_{A}\right)=a_{\mathrm{mem}}=m g_{\max } \frac{\tan \phi-\mu}{1+\mu \tan \phi} .
$$

When the gravitational force begins to decrease, all contacts are nonsliding. The displacements must be calculated using the matrix given in Eq. (37). The particle rises by a distance $m\left(g_{\max }-g_{*}\right) /(2 k)$, while $a_{0}$ remains constant, as in Eq. (38). The particle's position is now

$$
u_{y}=-\frac{m g_{*}+a_{\mathrm{mem}}}{2 k} .
$$

It is important to realize that the particle is $a_{\text {mem }} /(2 k)$ lower than it would be if the gravity had been increased monotonically from 0 to $g_{*}$. This displacement thus represents a "memory" of the forces exerted on disk in the past. But it is more convenient to think of the memory as being stored in $a_{0}$, for the displacement is proportional to $1 / k$ and very small if the particle is stiff, whereas $a_{0}$ is proportional to $g_{\max }$ and independent of the current value of $g$.

When the torque is applied, the contacts are still nonsliding, and they remain so until one of the contacts becomes 
sliding. We can determine which contact slides first by checking if $a_{\text {mem }}$ is greater than or less than $a_{2}$, with $a_{2}$ given in Eq. (17). If $a_{\mathrm{mem}}<a_{2}$, then the system meets the lower side of the funnel first, meaning contact $\alpha$ slides. Otherwise, it meets the upper edge first, and contact $\beta$ slides. Using Eqs. (17) and (44), we obtain that $a_{\mathrm{mem}}>a_{2}$ is equivalent to

$$
g_{\max }>g_{*}\left(1+\frac{\mu}{\tan \phi} \frac{1+\tan ^{2} \phi}{1+\mu^{2}}\right) .
$$

If this condition holds, then as $\tau$ increases, $a_{0}$ decreases to maintain equality in Eq. (9c). Eventually, equality is obtained in Eq. (9a), i.e., contact $\alpha$ begins to slip as well, and the disk rotates. This occurs when $a_{0}=a_{2}$. On the other hand, if Eq. (46) is not obeyed, than contact $\alpha$ slips first. Then $a_{0}$ increases until $a_{0}=a_{2}$, and the disk begins to rotate. Evaluating Eq. (46) for $\mu=0.5, \phi=50^{\circ}$, one obtains $g_{\max } \approx 1.8 g_{*}$, consistent with Fig. 5, where simulations with $g_{\max } / g$ $=2,4,6,8$ all meet the upper edge of the funnel. As in Sec. IV D 2, the motion occurs through a mechanism. This can be shown by constructing $\mathbf{Q}$, and showing that $\mathbf{Q} \mathbf{v}_{*}=0$ for $\mathbf{v}_{*}^{T}$ $=(0,0,1)$. Note that $\mathbf{v} *$ here corresponds to the disk rotating in place.

\section{Steep slopes: $\tan \phi>1 / \mu$}

For $\tan \phi>1 / \mu$, all the calculations in the previous section apply. The only difference is that the slope of the line given by equality in Eq. (9c) changes sign. This line is shown in Fig. 6, and if the system were to behave as before, it would follow this line upward indefinitely, leading to an infinite torque. However, the simulations show otherwise. When Eq. (9c) is attained, the system suddenly jumps to the rotating state, in a behavior qualitatively different from the other cases. This is an example of motion through an instability. The jump occurs when $T_{\beta}=-\mu R_{\beta}$, so this suggests that we ought to calculate the displacements of the disk when contact $\beta$ is sliding. Calculating $k \mathbf{a S c}^{T}$ as before, one obtains

$$
\mathbf{v}=\frac{\dot{\tau}}{2 k} \frac{\csc \phi}{(1-\mu \tan \phi)}\left(\begin{array}{c}
\mu-\tan \phi+\sec \phi \csc \phi \\
\tan \phi(\mu+\tan \phi) \\
(1 / r) \sec ^{2} \phi \csc \phi
\end{array}\right) .
$$

Now let us compute the $\dot{\mathbf{f}}_{\mathrm{ext}} \cdot \mathbf{v}$. We have $\dot{\mathbf{f}}_{\mathrm{ext}}=(0,0, \dot{\tau})^{T}$, so

$$
\dot{\mathbf{f}}_{\mathrm{ext}} \cdot \mathbf{v}=\frac{\dot{\tau}^{2}}{2 k r}\left(\frac{\csc ^{2} \phi \sec ^{2} \phi}{1-\mu \tan \phi}\right) \text {. }
$$

All factors on the right hand side are positive, except 1 $-\mu \tan \phi$ in the denominator, which is positive when $\tan \phi$ $<1 / \mu$ and negative when $\tan \phi>1 / \mu$. Thus for steep slopes, the condition $\dot{\mathbf{f}}_{\text {ext }} \cdot \mathbf{v}>0$ given in Sec. IV C is violated. The quasistatic assumption no longer holds.

Let us pause for a moment to consider what $\dot{\mathbf{f}}_{\text {ext }} \cdot \mathbf{v}$ $=\mathbf{v}^{T} \mathbf{Q} \mathbf{v}<0$ means in this case. Applying a positive torque to the disk means trying to rotate it in the positive direction, i.e., trying to impose $\omega>0$. Now Eq. (47) gives the velocities necessary to cancel the increasing external torque $\tau$. When $\tan \phi<1 / \mu$ (intermediate slopes), Eq. (47) shows that motion needed to do this requires rotation in positive direction. Therefore, the configuration is stable: when $\tau$ increases, the particle rotates in the positive direction, and the contact forces balance the increased torque. On the other hand, when $\tan \phi>1 / \mu$, the particle must be rotated in the negative direction to balance an increasing torque. Rotating the particle in the positive direction reduces the forces opposing the torque, and the disk accelerates, leading to a rapid change in the contact forces.

We can now predict the yielding torque in the MD simulations. If Eq. (46) is not satisfied, the system meets the lower edge of the funnel that functions normally, and the disk rotates when $\tau=\tau_{2}$. An example of this is the MD simulation at $g_{\max } / g=2$ in Fig. 6. On the other hand, if Eq. (46) is satisfied, the system meets the other branch of the funnel, and immediately starts to rotate. We can calculate the torque where this happens because the value of $a_{0}$ is known. Inserting the value of $a_{0}$ given in Eq. (44) into Eq. (9c) and solving for the torque gives

$$
\tau=m r \sin \phi\left[g_{*}+g_{\max }\left(\frac{\tan \phi-\mu}{\tan \phi+\mu} \frac{\mu \tan \phi-1}{\mu \tan \phi+1}\right)\right] .
$$

This result accurately predicts the yielding torque, as shown in Fig. 7.

\section{CONCLUSIONS}

From these investigations, we have shown that there are two ways that motion can begin.

(1) The first way ("motion through a mechanism") occurs when $\tan \phi<1 / \mu$. The set of solutions to equations of mechanical equilibrium [Eq. (1) or Eq. (7)] which also satisfy the contact conditions [Eq. (8)] becomes empty. The onset of motion can be predicted under the approximation of rigid particles (Sec. II C), and the CD and MD simulations give identical predictions. When $\mu<\tan \phi<1 / \mu$, these two simulation methods give different predictions for the contact forces, but this difference is irrelevant to the onset of motion. At the onset of motion, the contact forces change slowly, as they do before the motion begins.

(2) The second way ("motion through an instability") occurs when $\tan \phi>1 / \mu$. The onset of motion is through an instability, and cannot be predicted under the assumption of perfectly rigid particles. CD and MD now give differing predictions. At the onset of motion, the contact forces jump, changing much more rapidly than the applied forces do.

The methods used to predict the onset of motion can be easily generalized to assemblies of many particles. Therefore, we expect that these two ways to motion will exist in many particle systems.

This work also showed that there is a relation between memory and indeterminacy. When particles are assumed to be rigid, the forces cannot be uniquely determined. A certain combination of forces, whose amplitude is given by the coefficient $a_{0}$ in Eq. (6), causes no acceleration, and thus cannot be deduced by examining the equations of static equilibrium. However, its value can be constrained by enforcing Coulomb friction and the absence of cohesion at all the contacts. On the other hand, when the particles 
are assumed to be deformable, $a_{0}$ contains information about the history of the packing. This finding suggests that methods that average over different possible force networks [2] are really averaging over past histories of the packing.

Finally, this work demonstrated that giving the CD iterative solver the solution of the last time step as the initial guess is not just an innocent numerical trick. It is an essential part of the algorithm that represents a real physical process at work in granular material, namely, the memory of the packing encoded in the combinations of force that cause no acceleration. In certain situations, this memory could have an important effect on the behavior of the packing.

It is hoped that the findings presented in this paper will deepen and clarify our understanding of the quasistatic deformation of much larger packings. When the number of par- ticles is larger, there is not just one undetermined combination of forces, but many. However, one can expect that the effects studied here will be present in these more complicated situations, perhaps combined with each other in new and unexpected ways.

\section{ACKNOWLEDGMENTS}

The authors want to acknowledge the EU project "Degradation and Instabilities in Geomaterials with Application to Hazard Mitigation" (DIGA) in the framework of the Human Potential Program, Research Training Networks (HPRN-CT2002-00220). The authors wish to thank F. AlonsoMarroquín and J. J. Moreau for encouragement and interesting discussions.
[1] F. Radjai, L. Brendel, and S. Roux, Phys. Rev. E 54, 861 (1996).

[2] J. H. Snoeijer, T. J. H. Vlugt, M. van Hecke, and W. van Saarloos, Phys. Rev. Lett. 92, 054302 (2004).

[3] T. Unger, J. Kertész, and D. E. Wolf (unpublished).

[4] S. McNamara and H. Herrmann, Phys. Rev. E 70, 061303 (2004).

[5] J. J. Moreau, in Powders and Grains 2005, edited by S. McNamara, R. Garcia-Rojo, H. J. Hermann (Balkema, Rotterdam, 2005).

[6] T. C. Halsey and D. Ertas, Phys. Rev. Lett. 83, 5007 (1999).

[7] J. J. Moreau, in Novel Approaches in Civil Engineering, edited by M. Frémond and F. Maceri, Lecture Notes in Applied and
Computational Mechanics, Vol. 14 (Springer, New York, 2004), p. 1.

[8] P. A. Cundall and D. L. Strack, Geotechnique 29, 47 (1979).

[9] J.-N. Roux, Phys. Rev. E 61, 6802 (2000).

[10] Y. Kishino, H. Akaizawa, and K. Kaneko, in Powders and Grains 2001, edited by Y. Kishino (Balkema, Rotterdam, 2001).

[11] J.-N. Roux and G. Combe, C. R. Phys. 3, 131 (2002).

[12] R. Hill, The Mathematical Theory of Plasticity (Clarendon Press, Oxford, 1950).

[13] D. Kolymbas, Introduction to Hypoplasticity (Balkema, Rotterdam, 2000).

[14] R. Hill, J. Mech. Phys. Solids 6, 236 (1958). 